\title{
Interleukin-8 released by cancer-associated fibroblasts attenuates the autophagy and promotes the migration of ovarian cancer cells
}

\author{
SUYANEE THONGCHOT ${ }^{1,2}$, PRANISA JAMJUNTRA ${ }^{1}$, SUWANIT THERASAKVICHYA ${ }^{3}$, MALEE WARNNISSORN $^{4}$, \\ ALESSANDRA FERRARESI $^{5}$, PETI THUWAJIT $^{1}$, CIRO ISIDORO $^{5}$ and CHANITRA THUWAJTT $^{1}$ \\ ${ }^{1}$ Department of Immunology; ${ }^{2}$ Siriraj Center of Research Excellence for Cancer Immunotherapy; \\ Departments of ${ }^{3}$ Gynecology and Obstetrics, and ${ }^{4}$ Pathology, Faculty of Medicine Siriraj Hospital, \\ Mahidol University, Bangkok 10700, Thailand; ${ }^{5}$ Laboratory of Molecular Pathology, \\ Department of Health Sciences, University of Eastern Piedmont 'A. Avogadro', I-28100 Novara, Italy
}

Received August 20, 2020; Accepted February 12, 2021

DOI: $10.3892 /$ ijo.2021.5194

\begin{abstract}
The tumor microenvironment composed of a mixture of stromal cells and their secretions has a marked impact on cancer progression. In particular, soluble factors and metabolites contribute to malignancy through the dysregulation of autophagy in cancer cells. The present study investigated the effects of ovarian cancer-associated fibroblasts (OVCAFs) with their secretory substances on the autophagy and migration of ovarian cancer cells. The conditioned-medium (CM) of OVCAFs isolated from fresh human ovarian cancer tissues was analyzed for the levels of 27 common cytokines/chemokines using a cytokine array. Autophagy in cancer cells was assessed by determining the expression of the vacuolar form of LC3
\end{abstract}

Correspondence to: Dr Chanitra Thuwajit, Department of Immunology, Faculty of Medicine Siriraj Hospital, Mahidol University, 2 Wangluang, Siriaj, Bangkok 10700, Thailand

E-mail: chanitra.thu@mahidol.ac.th

Professor Ciro Isidoro, Laboratory of Molecular Pathology, Department of Health Sciences, University of Eastern Piedmont 'A. Avogadro', Via P. Solaroli 17, I-28100 Novara, Italy

E-mail: ciro.isidoro@med.uniupo.it

Abbreviations: $\alpha$-SMA, $\alpha$-smooth muscle actin; CAFs, cancer-associated fibroblasts; OVCAF-CM, conditioned-medium of cancer-associated fibroblasts; CK19, cytokeratin 19; FAP, fibroblast activation protein; IL, interleukin; bFGF, basic fibroblast growth factor; G-CSF, granulocyte-colony stimulating factor; GM-SCF, granulocyte-monocyte-colony stimulating factor; IFN- $\gamma$, interferon- $\gamma$; IP-10, interferon- $\gamma$-induced protein 10; MCP-1, monocyte chemoattractant protein-1; MIP-1 $\alpha$, monocyte inhibiting protein-1 $\alpha$; MIP-1 $\beta$, monocyte inhibiting protein-1 $\beta$; NFs, normal fibroblasts; PDGF-BB, platelet-derived growth factor-BB; RANTES, regulated on activation, normal $\mathrm{T}$ cell expressed and secreted; TNF- $\alpha$, tumor-necrosis factor- $\alpha$; VEGF, vascular endothelial growth factor; VIM, vimentin

Key words: ovarian cancer, autophagy, IL-8, cancer-associated fibroblast, migration, cytokines, tumor microenvironment by western blot analysis and immunofluorescence. Cancer cell migration was assessed by Transwell migration assay. Interleukin (IL)-8 was found to be the most highly upregulated cytokine among the cytokines/chemokines found in the OVCAF-CM. The role of IL-8 in ovarian cancer cell migration and its mechanistic link with autophagy was investigated. Recombinant human IL-8 (rhIL-8) stimulated the migration of SKOV3 and Kuramochi ovarian cancer cells, and concurrently downregulated basal autophagy, in concentration-dependent manner. Compared to the CM of control counterpart normal fibroblasts isolated from benign ovaries (OVNF-CM), the CM from 3 OVCAF isolates (namely, OVCAF-9, -20 and -43) exerted effects similar to rhIL-8 on both cancer cell lines. The pharmacological induction of autophagy with rapamycin or metformin attenuated the pro-migratory effects of IL-8. Neutralizing anti-IL-8 antibody counteracted the inhibitory effect of OVCAF-CM on basal autophagy. On the whole, the present study highlights the involvement of IL- 8 released by CAFs in the ovarian tumor microenvironment in promoting cancer cell migration through the suppression of autophagy.

\section{Introduction}

Ovarian cancer is the third most common gynecological malignancy (1). Improvements in chemotherapeutics have only resulted in modest increases in the survival rates. A large majority of patients is diagnosed at an advanced stage of the disease (stage III or IV), often with bowel obstruction and systemic involvement, which explains the high lethality of this type of tumor among women (2). At this stage, patients present with widespread metastatic growth within the peritoneal cavity, where the ascitic fluid enriched of growth factors and inflammatory cytokines further contributes to the growth and dissemination of cancer cells.

Cancer-associated fibroblasts (CAFs) in the tumor microenvironment have been well-recognized for their potential to induce cancer cell progression in ovarian cancer $(3,4)$. Ovarian CAFs secrete a variety of soluble factors that have been shown to affect the metabolism and the phenotypes of ovarian cancer cells, and in this respect interleukin (IL)- 8 is one of the cytokines playing a major role in this process (3). 
The serum levels of IL-8 (and IL-10) closely parallel the stage and prognosis of ovarian cancer (5). Additionally, the concentration of IL-8 (and of IL-6) in the peritoneal fluid increases along with ovarian cancer progression and represents a factor of poor prognosis (6). At the biological level, IL-8 has been shown to induce ovarian cancer cell migration, invasion and epithelial-mesenchymal transition (EMT) (7-9), and to promote the growth of ovarian cancer 3-dimensional spheroids (10). Understanding the mechanisms underlying ovarian cancer cell migration is challenging, yet it is instrumental for designing effective therapeutic strategies that could prevent the metastatic spreading of the tumor and improve the prognosis of patients.

Autophagy, a lysosomal catabolic process with homeostatic and pro-survival functions, influences the behavior of ovarian cancer cells, affecting a variety of processes, such as survival in metabolic harsh conditions, invasive growth, the development of immune- and chemo-resistance, the maintenance of stem-like properties and dormancy $(3,11)$. Autophagy is dysregulated in ovarian cancer $(12,13)$, and the autophagy-related proteins BECLIN-1 and LC3 are in fact prognostic factors for ovarian cancer (14-17). The migration rate of ovarian cancer cells increases upon autophagy gene knockdown (13). Several cytokines/chemokines from ovarian CAFs have been proven to modulate autophagy (3). The authors have previously demonstrated that IL-6 downregulates autophagy in ovarian cancer cells, while promoting their motility (18). IL-8 has been shown to interrupt the dormant state of ovarian cancer cells by attenuating DIRAS3-mediated autophagy (19). IL-8 has been shown to induce EMT in ovarian cancer cells and to enhance ovarian cancer cell metastasis (9). Consistently, in vitro studies have indicated that the overexpression and secretion of IL-8 in ovarian cancer cells favor their anchorage-independent growth, proliferation and invasion (20). However, to date there are no data available showing a direct effect of IL- 8 secreted by ovarian cancer CAFs on the modulation of autophagy and how this modulation affects ovarian cancer cell migration. The present study aimed to provide knowledge on this matter. To this end, primary cultured ovarian CAFs (OVCAFs) were isolated from fresh surgical ovarian cancer tissues and their secreted substances in the conditioned-media (OVCAF-CM) were characterized. To the best of our knowledge, the present study demonstrates for the first time that IL- 8 is a major cytokine driving ovarian cancer cell migration and that this effect is mechanistically linked to the downregulation of autophagy in cancer cells. The present findings indicate IL- 8 as a therapeutic target (e.g., with recombinant specific antibody) to hinder its activity and restore autophagy in cancer cells, and by so doing prevent the metastatic spreading of ovarian cancer.

\section{Materials and methods}

Human ovarian cancer cell lines and cell culture. The human ovarian cancer cell lines, SKOV3 (ATCC, Cell Systems \& cGMP Biorepository) and Kuramochi (Japanese Collection of Research Bioresources), were employed in the present study. The SKOV3 cells and Kuramochi cells were grown in Dulbecco's modified Eagle's medium (DMEM; Gibco; Thermo Fisher Scientific, Inc.) and RPMI-1640 (Gibco; Thermo Fisher
Scientific, Inc.), respectively. Culture media were supplemented with $10 \%(v / v)$ fetal bovine serum (FBS) (Gibco; Thermo Fisher Scientific, Inc.) and 1\% penicillin/streptomycin (Gibco; Thermo Fisher Scientific, Inc.). Cells were maintained in an incubator at $37^{\circ} \mathrm{C}$ with $5 \% \mathrm{CO}_{2}$. Cells at a passage of $<10$ and exhibiting $>90 \%$ viability (measured by trypan blue staining, data not shown) were used in the experiments.

Primary culture of OVCAFs. Ovarian cancer tissues were obtained from patients undergoing oophorectomy at Siriraj Hospital, Mahidol University. Ethical approval was granted by the Siriraj Institution Board (COAs no. si670/2013 and si246/2017). The patients released the informed consent for the usage of their samples for research purposes in written form. The samples were collected at Siriraj Hospital (Bangkok) during the period of 2013-2019. Tissue sections of approximately $10 \mathrm{~mm}^{3}$ were submerged in $2 \mathrm{X}$ antibiotic-containing DMEM with $10 \%$ FBS overnight at $4^{\circ} \mathrm{C}$ to reduce microbial contamination. The sample was rinsed with $1 \mathrm{X}$ phosphate-buffered saline (PBS) and cut into small sections of $2 \mathrm{~mm}^{3}$. Cell debris was washed out with $10 \%$ FBS-containing DMEM with $1 \mathrm{X}$ penicillin $(100 \mathrm{U} / \mathrm{ml})-$ streptomycin $(100 \mu \mathrm{g} / \mathrm{ml})(\mathrm{Gibco}$; Thermo Fisher Scientific Inc.), amphotericin B $(5 \mathrm{mg} / \mathrm{ml})$ (Amphotret $^{\mathrm{TM}}$, Bharat Serums and Vaccines Limited Ltd.) and HEPES (20 mM) (Gibco; Thermo Fisher Scientific Inc.) (complete medium). The tissue sections were then transferred and allowed to adhere to a Petri dish at $37^{\circ} \mathrm{C}$ in a $\mathrm{CO}_{2}$ incubator. After the fibroblast-like cells protruded from the underneath the tissues, adherent cells were trypsinized with $0.25 \%$ trypsin/EDTA (Gibco; Thermo Fisher Scientific Inc.) and re-plated. The complete medium was replaced every 2-3 days. Upon reaching confluency, the cells were trypsinized and re-plated. Cultures of fibroblasts at passages 4 to 17 were used in the experiments after confirming the CAF biochemical and morphological features. These cells were named OVCAFs. The normal-associated fibroblasts (OVNFs) were isolated with the same method from adjacent non-tumoral ovarian tissues of the same patient.

Characterization of primary culture of OVCAFs. Immunocytochemistry of cytokeratin 19 (CK19), $\alpha$-smooth muscle actin ( $\alpha$-SMA), vimentin (VIM) and fibroblast activation protein (FAP) was performed to verify the purity of OVCAFs and OVNFs. Briefly, cells on sterile glass coverslips were fixed in ice-cold methanol and then incubated overnight at $4^{\circ} \mathrm{C}$ in a humid chamber with the indicated primary antibodies as follow: 1:200 mouse anti-human CK-19 antibody (sc-6278, Santa Cruz Biotechnology Inc.), 1:500 anti- $\alpha-$ SMA antibody (A5228, Abcam), 1:500 anti-VIM antibody (sc-6260, Santa Cruz Biotechnology Inc.), and 1:500 rabbit anti-human FAP (ab53066, Abcam). After washing out the excess primary antibody, the coverslip was incubated for $3 \mathrm{~h}$ at room temperature with the appropriate secondary fluorescent antibody including goat anti-mouse IgG-Cy3 antibody $(1: 2,000$, \#115-166-071, Jackson ImmunoResearch Laboratories Inc.) or the goat anti-rabbit IgG-FITC antibody $(1: 2,000$, ab6717, Abcam). Hoechst 33342 solution (Invitrogen; Thermo Fisher Scientific, Inc.) was added to stain the nucleus. Fluorescence was captured using an Inverted microscope model IX71 (Olympus Corporation). 
Conditioned-media (CM) collection from fibroblasts. OVCAFs and OVNFs were cultured in $75-\mathrm{cm}^{2}$ flasks to reach 90-95\% confluency in DMEM containing 10\% FBS. Cells were then washed twice with $1 \mathrm{X}$ PBS and twice with $1 \%(\mathrm{v} / \mathrm{v})$ FBS-DMEM and incubated for $24 \mathrm{~h}$ at room temperature. Subsequently, the CM were collected and designated as $24 \mathrm{~h}$ CM. CMs were centrifuged at $2,000 \mathrm{x} \mathrm{g}$ at $4^{\circ} \mathrm{C}$ for $5 \mathrm{~min}$ to remove cell debris and the suspension stored at $-80^{\circ} \mathrm{C}$ until use. The Bradford assay kit (Bio-Rad Laboratories, Inc.) was used to measure the amount of total protein and adjusted to be equal when supplemented to the cells.

Wound healing migration assay. The SKOV3 and Kuramochi cells were cultured in a 6 -well plate until they reached approximately $90 \%$ confluency. The reference lines were drawn at the middle under the plate. Cells were scratched using a sterile $1,000 \mu \mathrm{l}$ pipette tip followed by washing 3 times with serum-free medium to discard unattached cells. The cells were treated with either OVCAF-CM or OVNF-CM or with $0,25,250,2,500$ and 25,000 pg/ml recombinant human IL-8 (rhIL-8)/CXCL8 (11349086, ImmunoTools GmbH). Other treatments included anti-IL-8/CXCL8 neutralizing antibody (MAB208, R\&D Systems, Inc.), 200 nM rapamycin (R8781, Sigma-Aldrich; Merck KGaA) and 2 mM metformin hydrochloride (PHR1084, Sigma-Aldrich; Merck KGaA), as indicated. Incubations were performed in medium supplemented with 1 or $10 \%$ FBS, as indicated. The scratched areas indicated by the reference line were recorded at the beginning and $24 \mathrm{~h}$ after treatment. The migration efficiency was determined as the percentage of healing of the scratched area as calculated by the following formula: \% wound healing=wound space at $0 \mathrm{~h}$-at the end of the experiment $\mathrm{x} 100 /$ wound space at $0 \mathrm{~h}$.

Two dimensional (2D) transwell Fluoroblok ${ }^{T M}$ migration assay. The ovarian cancer cell lines were cultured in $25-\mathrm{cm}^{2}$ flasks until they reached $80 \%$ confluency. Following trypsinization, the cells were fluorescently labeled by incubation with $5 \mu \mathrm{mol} / 1$ Green CMFDA Dye (Invitrogen; Thermo Fisher Scientific, Inc.) for $30 \mathrm{~min}$ following the manufacturer's protocol. The cells, $1 \times 10^{5}$ cells/well for SKOV3 and $2 \times 10^{5}$ cells/well for Kuramochi, were seeded onto the coated $8 \mu \mathrm{m}$ pore Fluoroblok ${ }^{\mathrm{TM}}$ membrane insert in the upper chambers of 24-well companion plates (BD Biosciences). OVCAF-CM or OVNF-CM was added to the cells in the upper chamber and into the lower chamber. Fluoroblok ${ }^{\mathrm{TM}}$ plates were incubated in a humidified atmosphere at $37^{\circ} \mathrm{C}$ in $5 \% \mathrm{CO}_{2}$ in the Synergy $\mathrm{H} 1$ instrument (Biotek Instruments, Inc.). The measurement of the relative fluorescence unit (RFU) at an excitation of $490 \mathrm{~nm} / \mathrm{emission} 520 \mathrm{~nm}$ was measured for the migrated cells. A total of 3 independent experiments were performed.

Western blot analysis of LC3 and p62 in ovarian cancer cells. The ovarian cancer cells were seeded in a 6-well plate and cultured for $24 \mathrm{~h}$ in complete medium with $10 \% \mathrm{FBS}$ as a negative control for autophagy, or $1 \%$ FBS containing medium as a positive control for autophagy, or in OVCAF-CM or OVNF-CM, as indicated. Chloroquine (CLQ; $30 \mu \mathrm{M}$ ) (Sigma-Aldrich; Merck KGaA) was added to CM $8 \mathrm{~h}$ prior to cell collection. The cells were harvested and washed with $1 \mathrm{X}$
PBS, and lysed with in-house made radioimmuno-precipitation assay (RIPA) buffer containing $150 \mathrm{mM} \mathrm{NaCl}, 1.0 \%$ IGEPAL $^{\circledR}$ CA-630, 0.5\% sodium deoxycholate, $0.1 \%$ SDS, $50 \mathrm{mM}$ Tris, $\mathrm{pH} 8.0$ and then heated for $5 \mathrm{~min}$ at $95^{\circ} \mathrm{C}$ to denature proteins. Protein samples were separated by $15 \%$ SDS-polyacrylamide gel electrophoresis (SDS-PAGE) and transferred onto polyvinylidene fluoride (PVDF) membranes by TE 70 Semi-Dry transfer units (GE Healthcare) for $1.5 \mathrm{~h}$. The membranes were blocked in a blocking solution containing $5 \%(\mathrm{w} / \mathrm{v})$ skim milk in $0.1 \%(\mathrm{v} / \mathrm{v})$ Tween 20 in TBS-T for $1 \mathrm{~h}$ at room temperature. The blots were probed by overnight incubation at $4^{\circ} \mathrm{C}$ with rabbit anti-human LC3B antibody (1:1,000, L7543, Sigma-Aldrich; Merck KGaA) or rabbit anti-human p62 antibody (1:500, ab109012, Abcam). The blots were then washed in TBS-T before being incubated with 1:2,000 goat anti-rabbit IgG-HRP (ab6721, Abcam) for $1 \mathrm{~h}$. For $\beta$-actin detection, mouse anti-human $\beta$-actin monoclonal antibody $(1: 5,000)$ (sc-47778, Santa Cruz Biotechnology Inc.) and 1:2,000 HRP-conjugated horse anti-mouse IgG antibody (7076, Cell Signaling Technology Inc.) in blocking solution were used as primary and secondary antibody, respectively. The protein bands were developed by adding Clarity ${ }^{\mathrm{TM}}$ Western ECL substrate (170-5061, Bio-Rad Laboratories, Inc.) and detected under Gel Document (Syngene ${ }^{\circledR}$ ). The band intensities were measured using ImageJ software version 1.52a. The level of $\beta$-actin was used as an internal control to ensure equal amounts of loading proteins.

Immunofluorescence detection of LC3 and of E-cadherin in cells at the migration front. The ovarian cancer cells were allowed adhere on sterile glass coverslips and cultured until they reached $>90 \%$ confluency. The cell monolayers were scratched using a $200 \mu \mathrm{l}$ tip and then washed several times with $1 \mathrm{X}$ PBS to remove the cell debris. OVCAF-CM and OVNF-CM were added to the cells for $24 \mathrm{~h}$. Finally, the cells were fixed in ice-cold methanol and then incubated overnight at $4^{\circ} \mathrm{C}$ in a humid chamber with anti-human LC3B antibody (1:100) (L7543, Sigma-Aldrich; Merck KGaA) and anti-human E-cadherin (2Q663, sc-71008, Santa Cruz Biotechnology, Inc.) After washing out the excess of primary antibody, the coverslip was incubated for $3 \mathrm{~h}$ at room temperature with the appropriate fluorescence conjugated secondary antibody goat anti-rabbit IgG-FITC antibody (1:2,000, ab6717, Abcam) and goat anti-mouse IgG-CyTM3 antibody (115-166-071, Jackson ImmunoResearch Laboratories, Inc.). Antibodies were diluted in $0.1 \%$ Triton $\mathrm{X}$ in $1 \mathrm{X}$ PBS containing $10 \%$ FBS. Hoechst 33342 solution (Invitrogen; Thermo Fisher Scientific, Inc.) was then added to stain the nuclei (30 $\mathrm{min}$ at room temperature). Fluorescence signals were captured with a Leica DMIRE2 confocal fluorescence microscope (Leica Microsystems AG) equipped with Leica Confocal Software v. 2.61.

Protein array analysis of OVCAF-derived secreted substances. The cytokines/chemokines secreted by the CAFs into the CM were assayed using a ProHuman Cytokine Standard 27-Plex assay, Group I (\#M50-0KCAF0Y, Bio-Rad Laboratories, Inc.), which monitors 27 human cytokines, including interleukin (IL)-1 $\beta$ (IL-1 $\beta$ ), IL-1 receptor $\alpha$ (IL-1ra), IL-2, IL-4, IL-5, IL-6, IL-7, IL-8, IL-9, IL-10, IL-12p70, IL-13, IL-15, IL-17A, eotaxin, basic fibroblast growth factor 
(bFGF), granulocyte-colony stimulating factor (G-CSF), granulocyte-monocyte-colony stimulating factor (GM-CSF), interferon- $\gamma$ (IFN- $\gamma$ ), interferon- $\gamma$-induced protein 10 (IP-10), monocyte chemoattractant protein-1 (MCP-1), monocyte inhibiting protein-1 $\alpha$ (MIP- $1 \alpha)$, monocyte inhibiting protein- $1 \beta$ (MIP-1 $\beta$ ), platelet-derived growth factor-BB (PDGF-BB), RANTES, tumor-necrosis factor- $\alpha($ TNF- $\alpha$ ) and vascular endothelial growth factor (VEGF). The detection process was performed following the instruction leaflet. Briefly, $24 \mathrm{~h} \mathrm{CM}$ from OVCAFs and OVNFs was centrifuged at 1,000 $\mathrm{x} \mathrm{g}$ for $10 \mathrm{~min}$ at $4^{\circ} \mathrm{C}$ and the concentration of cytokines/chemokines of each sample was determined using the Bio-Plex 200 system (Bio-Rad Laboratories, Inc.). Data were analyzed using Bio-Plex Manager ${ }^{\text {TM }}$ Software Version 4.0 (Bio-Rad Laboratories, Inc.). The cytokine concentration $(\mathrm{pg} / \mathrm{ml})$ represents the mean \pm SD of 3 independent experiments in triplicate.

Statistical analysis. All statistical analyses were performed using SPSS version 23 (SPSS Inc.). The values are expressed as the means \pm SD. Statistical significance was determined by one-way ANOVA with the Tukey's post hoc test when comparing multiple samples. The Student's t-test was used when comparing each sample to the normal control. A P-value $<0.05$ was considered to indicate a statistically significant difference.

\section{Results}

OVCAF-derived CM induces ovarian cancer cell migration and reduces autophagy. OVCAFs isolated from fresh ovarian cancer tissues from 3 patients exhibited positivity for the specific markers, including $\alpha$-SMA, VIM and FAP, while they were negative for the epithelial marker, CK19 (Fig. 1). As a further confirmation, the OVCAFs, but not the normal fibroblasts isolated from counterpart benign ovary tissues of the same patients (OVNFs), expressed FAP (Fig. 1). The ability of the CM from OVCAFs and from OVNFs isolated from the 3 patients to induce the migration of fluorescently0labeled ovarian cancer SKOV3 and Kuramochi cells through a porous membrane of the Transwell was assayed (as described in the Materials and methods). As a baseline, control medium supplemented with $1 \%$ FBS was employed to maintain the level of serum factors to the minimum. Cell migration stimulated by the OVCAF-CM was evidently greater than that stimulated by the OVNF-CM counterpart, with statistical significance at all incubation times tested (Fig. 2A and B).

Given the role of autophagy in ovarian cancer cell migration $(18,21)$, the present study assessed the level of cellular autophagy in the treated cancer cells. The cells were incubated with medium as indicated in the presence of CLQ to prevent autophagosome degradation (22), and the expression of the autophagy marker, LC3, was analyzed in the homogenates by western blot analysis. Upon the induction of autophagy, the cytosolic LC3-I isoform is converted into the autophagosomal LC3-II isoform (22). The LC3-II/LC3-I ratio, considered a marker of the induction of autophagy (22), was decreased in both ovarian cancer cell lines exposed to OVCAF-CM (from 3 patients) compared to the OVNF-CM of the respective patients (Fig. 2C and D). The downregulation of autophagy in the SKOV3 cells was significant with the CM of OVCAF-9 and of OVCAF-20, whereas in the Kuramochi cells, the CM from OVCAF-20 was more effective. Notably, the cancer cells cultured in control culture medium containing $10 \%$ FBS exhibited a lower level of autophagy as compared to the cells cultured with 1\% FBS (Fig. 2C and D). Taken together, these results indicate a link between low autophagy and a high migration rate, and suggest that OVCAFs-CM induce ovarian cancer cell migration by reducing autophagy in the cancer cells. To confirm this link, the expression of vacuolar LC3 (corresponding to the autophagosomal LC3-II isoform) and of the epithelial marker E-cadherin (E-cad) was assessed by immunofluorescence staining in the ovarian cancer cells at the migration front. It is expected that E-cad, which mediates cell-cell adhesion, would be downregulated in cells that begin to migrate.

In both the SKOV3 and Kuramochi cells, incubation OVCAF-CM led to a reduced positivity for vacuolar LC3 and for membrane E-cad compared to the cells incubated with the counterpart OVNF-CM (Fig. 2E and F). This pattern is characteristic of migratory cells $(18,21)$. Serum factors are known to stimulate cell proliferation and cell migration (22) and to downregulate basal autophagy (23). Therefore, the present study assayed the expression of these proteins in cultures incubated in standard culture medium supplemented with low (1\%) or high (10\%) FBS as controls. A high LC3 and high E-cad expression pattern was observed in the cells cultured with $1 \%$ FBS medium and exhibiting a low invasive capability (Fig. 2A-D), whereas the opposite pattern (low LC3 and low E-cad expression) was observed in the cells cultured in $10 \%$ FBS medium exhibiting migratory features (Fig. 2E, F). These data consistently demonstrate that cancer cell migration induced by OVCAF-CM and by high serum (10\% FBS) is associated with the downregulation of autophagy.

OVCAF-derived secretion contains higher amounts of IL-8 compared to the OVNF counterpart. Secreted substances in OVCAF-CM and in OVNF-CM from 6 patients (case nos. 9, 20, 26, 34, 43 and 48) were assayed using a cytokine array for 27 cytokines/chemokines including IL-1 $\beta$, IL-1r $\alpha$, IL-2, IL-4, IL-5, IL-6, IL-7, IL-8, IL-9, IL-10, IL-12p70, IL-13, IL-15, IL-17A, eotaxin, bFGF, G-CSF, GM-CSF, IFN- $\gamma$, IP-10, MCP-1, MIP-1 $\alpha$, MIP-1 $\beta$, PDGF-BB, RANTES, TNF- $\alpha$ and VEGF. The raw data of the cytokine levels are presented in Table I. In total, 10 cytokines, namely IL-6, IL-8, eotaxin, GM-CSF, IFN- $\gamma$, IP-10, MCP-1, MIP-1b, RANTES and VEGF, were in the detectable range. Of the 6 OVCAF-CM, only 7 from the 10 total detectable cytokines, including IL-6, IL-8, G-CSF, IFN- $\gamma$, IP-10, MCP-1 and VEGF, exhibited a significantly increased level (P-value $<0.05$ and $<0.01$ ) (Fig. S1). All 6 OVCAF-CM exhibited statistically significant higher levels of IL-8 (P-value <0.01) and MCP-1 (P-value <0.05) compared with the 6 pooled OVNF-CM (Fig. 3A). Since a different genetic background can influence the gene expression and the secretion of cytokines, the matched OVNF-CM and OVCAF-CM from the same patients were selected and compared. The following were included in the assay: OVCAF-9 and OVNF-9, OVCAF-20 and OVNF-20, and OVCAF-43 and OVNF-43. The results revealed a significantly higher level of IL-8 in all 3 pairings of OVCAF-CM compared to OVNF-CM. In addition, the GM-CSF, IFN- $\gamma$, IP-10 and MCP-1 levels were increased in 

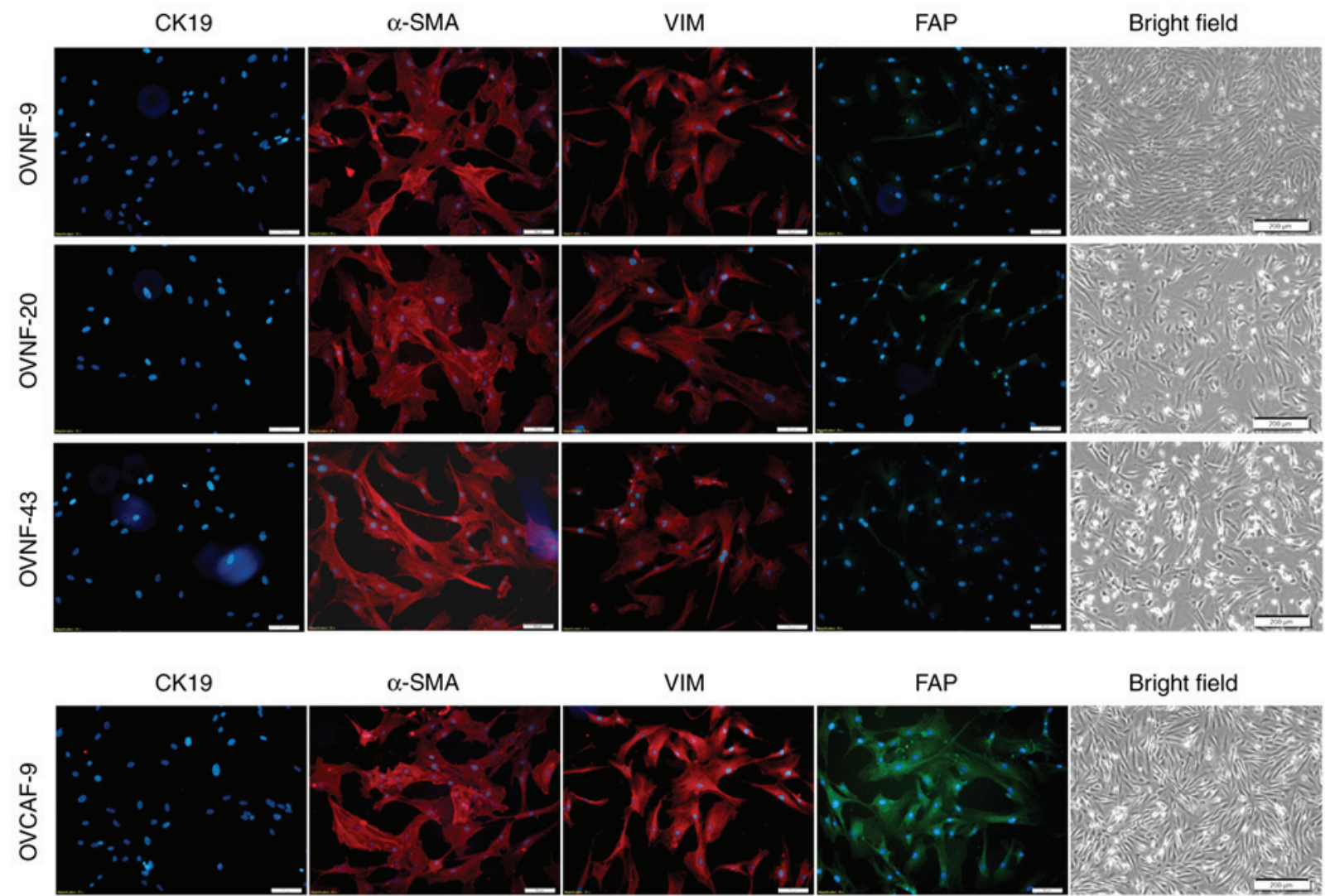

Bright field
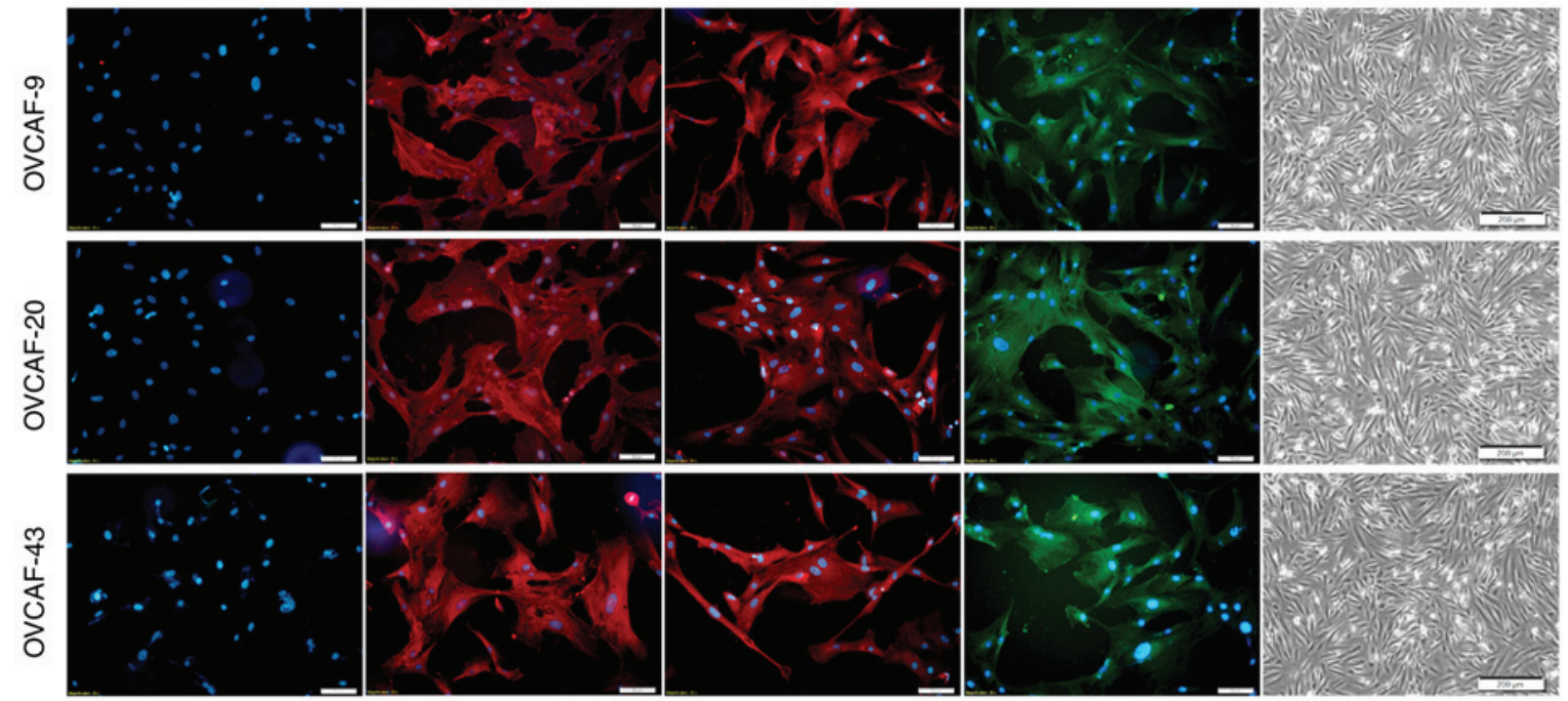

Figure 1. Quality control of the purity of OVNFs and OVCAFs. Fibroblasts were isolated from ovarian cancer tissues (OVCAF) and from adjacent healthy ovarian tissues (paired OVNFs) and assayed for purity by immunofluorescence using antibodies directed to specific markers for myofibroblasts ( $\alpha$-SMA, red fluorescence), epithelial cells (CK19, red fluorescence), fibroblast intermediate filament (VIM, red fluorescence) and fibroblast-associated protein (FAP, green fluorescence). The nuclei were counterstained with Hoechst. The unstained cells of each cell type are shown. All images shown in the figure are representative of 3 independent experiments. Scale bar, $50 \mu \mathrm{M}$. OVNFs, ovarian non-tumor-associated fibroblasts; OVCAFs, ovarian cancer-associated fibroblasts; $\alpha$-SMA, $\alpha$ smooth muscle actin; CK19, cytokeratin 19; FAP, fibroblast activation protein.

OVCAF-43-CM compared to those in the normal counterpart with statistical significance (Fig. 3B-D).

IL-8 promotes ovarian cancer cell migration and reduces autophagy. According to the consistent finding of high IL-8 in all OVCF-CM compared to their normal counterpart $\mathrm{CM}$, it was hypothesized that IL-8 may be the most relevant secreted substance in OVCAF-CM driving cell migration in association with the suppression of autophagy. To examine this hypothesis, the effect of rhIL-8 was assayed. The positive control of high basal autophagy was detected in cells cultured with $1 \%$ FBS containing medium compared to that in the cells grown in $10 \%$ FBS. CLQ was added to discriminate LC3-II accumulation arising from increased autophagosome formation from that due to impaired autophagosome degradation (24). As shown by the results of western blot analysis of the LC3-II/LC3-I ratio, it was evident that rhIL-8 significantly inhibited basal autophagy both in SKOV3 cells (at $25 \mathrm{pg} / \mathrm{ml}$ to $25,000 \mathrm{pg} / \mathrm{ml}$ ) and in Kuramochi cells (at 25,000 pg/ml) (LC3, Fig. 4A and B). The p62/SQSTM1 protein, a receptor for cargo destined to be degraded by autophagy, accumulates in cells when the autophagic flux is hampered (24). In the present study, p62 protein significantly accumulated in the cells treated with high concentrations of rhIL-8 (2,500 and $25,000 \mathrm{pg} / \mathrm{ml}$ ) (p62, Fig. 4A and B). Taken together, the results with both autophagy markers (LC3 and p62) support the conclusion that rhIL- 8 decreases the level of basal autophagy in ovarian cancer cells. 
A

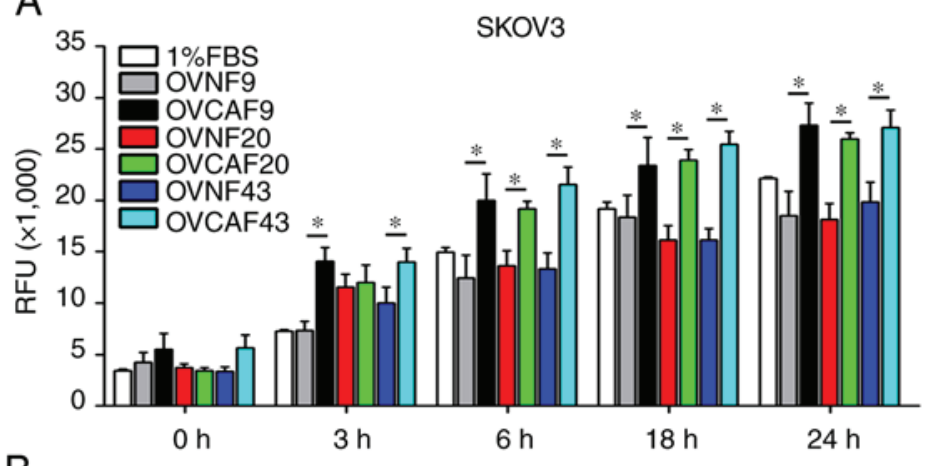

B

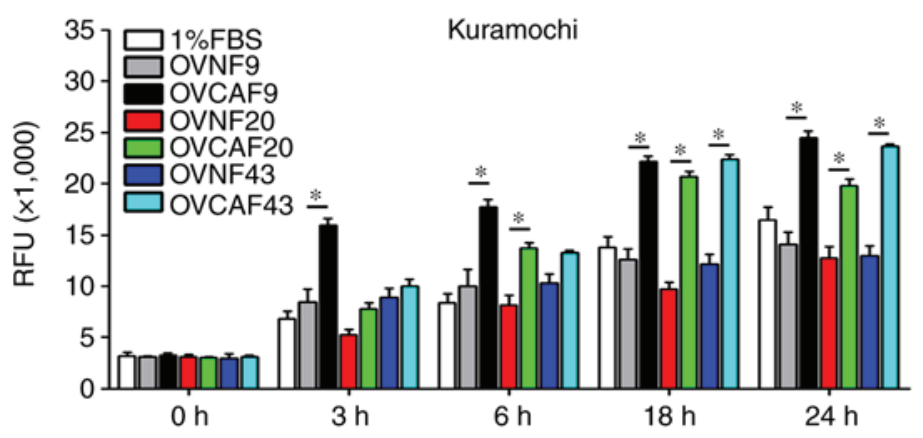

C
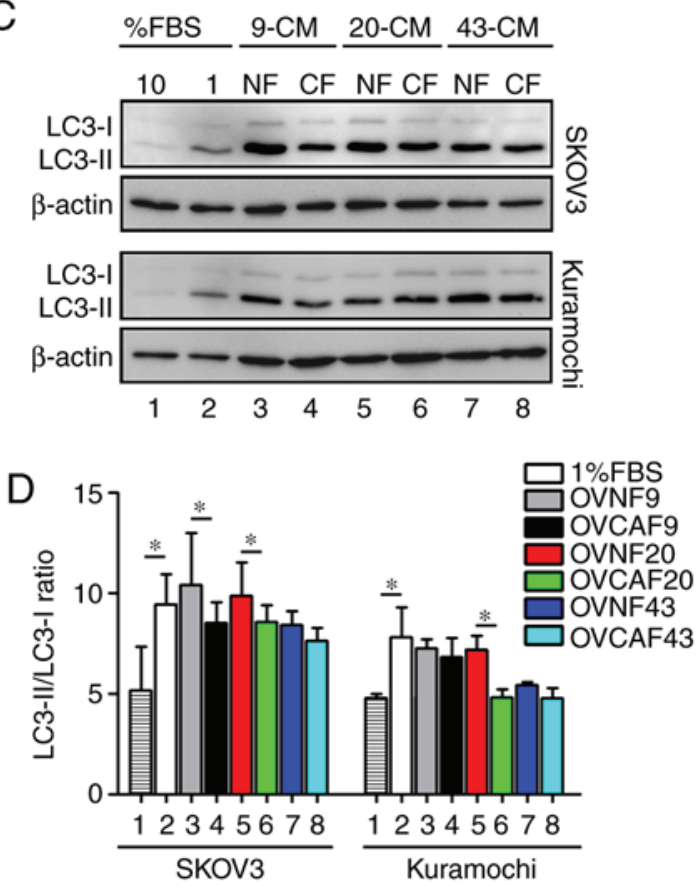

$\mathrm{E}$

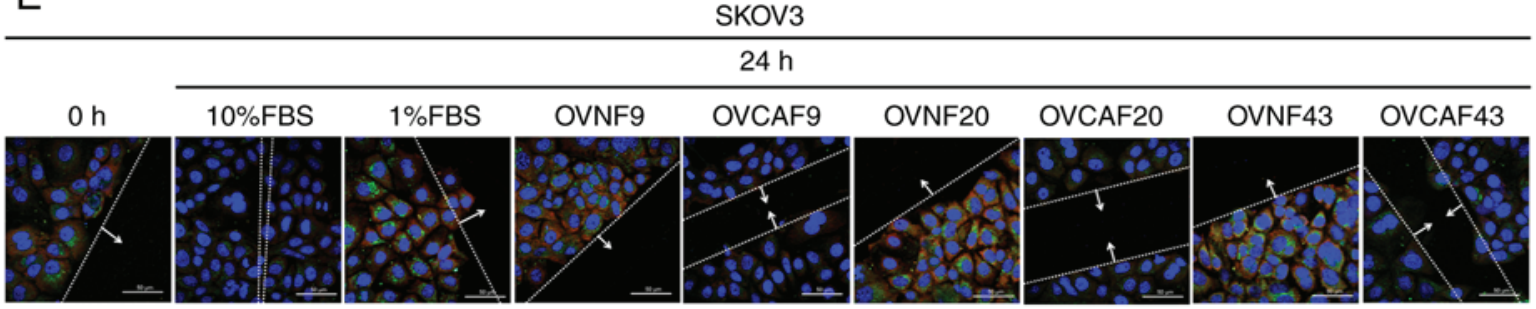

LC3/E-cad/Hoechst

$\mathrm{F}$ Kuramochi

\begin{tabular}{|c|c|c|c|c|c|c|c|c|}
\hline \multicolumn{9}{|c|}{$24 \mathrm{~h}$} \\
\hline $\mathrm{Oh}$ & $10 \%$ FBS & $1 \%$ FBS & OVNF9 & OVCAF9 & OVNF20 & OVCAF20 & OVNF43 & OVCAF43 \\
\hline & & & & & & & & \\
\hline
\end{tabular}

LC3/E-cad/Hoechst

Figure 2. OVCAF-derived CM induced ovarian cancer cell migration and reduced autophagy. (A and B) Representative RFUs of SKOV3 and Kuramochi migrated cells. The cells were seeded in 24-Fluoroblok ${ }^{\mathrm{TM}}$ membrane Transwell plates and treated with OVNFs-CM or OVCAFs-CM. The graphs report the rate of migration cells for each time point $(0,3,6,18$ and $24 \mathrm{~h})$. (C) Western blot analysis showing the level of LC 3 expressed by SKOV3 and Kuramochi cells exposed to OVNFs-CM or OVCAFs-CM, compared with 10\% FBS and 1\% FBS control media and in the presence of $30 \mu \mathrm{M}$ CLQ (added $8 \mathrm{~h}$ before cell lysis). (D) Densitometry of the bands is reported as the ratio of LC3-II/LC3-I. Bar graphs represent mean \pm SD of 3 independent experiments. Student's t-test showing the significant differences of OVCAF compared to normal fibroblasts, "P<0.05. (E and F) Double immunostaining for LC3 (green fluorescence) and E-cad (red fluorescence). Images show the co-localization of LC3 with E-cad in cells at the migration front under OVNFs-CM or OVCAFs-CM-treatment compared with 10\% FBS and 1\% FBS media control and in the presence of $30 \mu \mathrm{M} \mathrm{CLQ}$. The cells at the migration front in the wound area were photographed under the confocal fluorescence microscope. Scale bar, $50 \mu \mathrm{M}$; magnification, x63. RFU, relative fluorescence unit; LC3, light chain 3; E-cad, E-cadherin; OVNFs, ovarian non-tumor-associated fibroblasts; OVCAFs, ovarian cancer-associated fibroblasts.

To further support this contention, neutralizing anti-IL8 antibody was applied to the culture medium supplemented with FBS or rhIL-8. The addition of anti-IL8 to the culture medium restored the autophagic flux, as evidenced by the high LC3-II/LC3-I ratio and the reduced accumulation of p62, both in the SKOV3 and Kuramochi cells cultured in the presence of rhIL-8 or 10\% FBS (Fig. 4C and D). Immunofluorescence staining of LC3 further confirmed that IL-8 effectively inhibited autophagy in ovarian cancer cells (Fig. 4E). Of note, the fact that anti-IL-8 restored autophagy in the cells cultured with $10 \%$ FBS to the level of that in control cells cultured with $1 \%$ FBS suggests that FBS contains some IL-8 (Fig. 4C and D, 


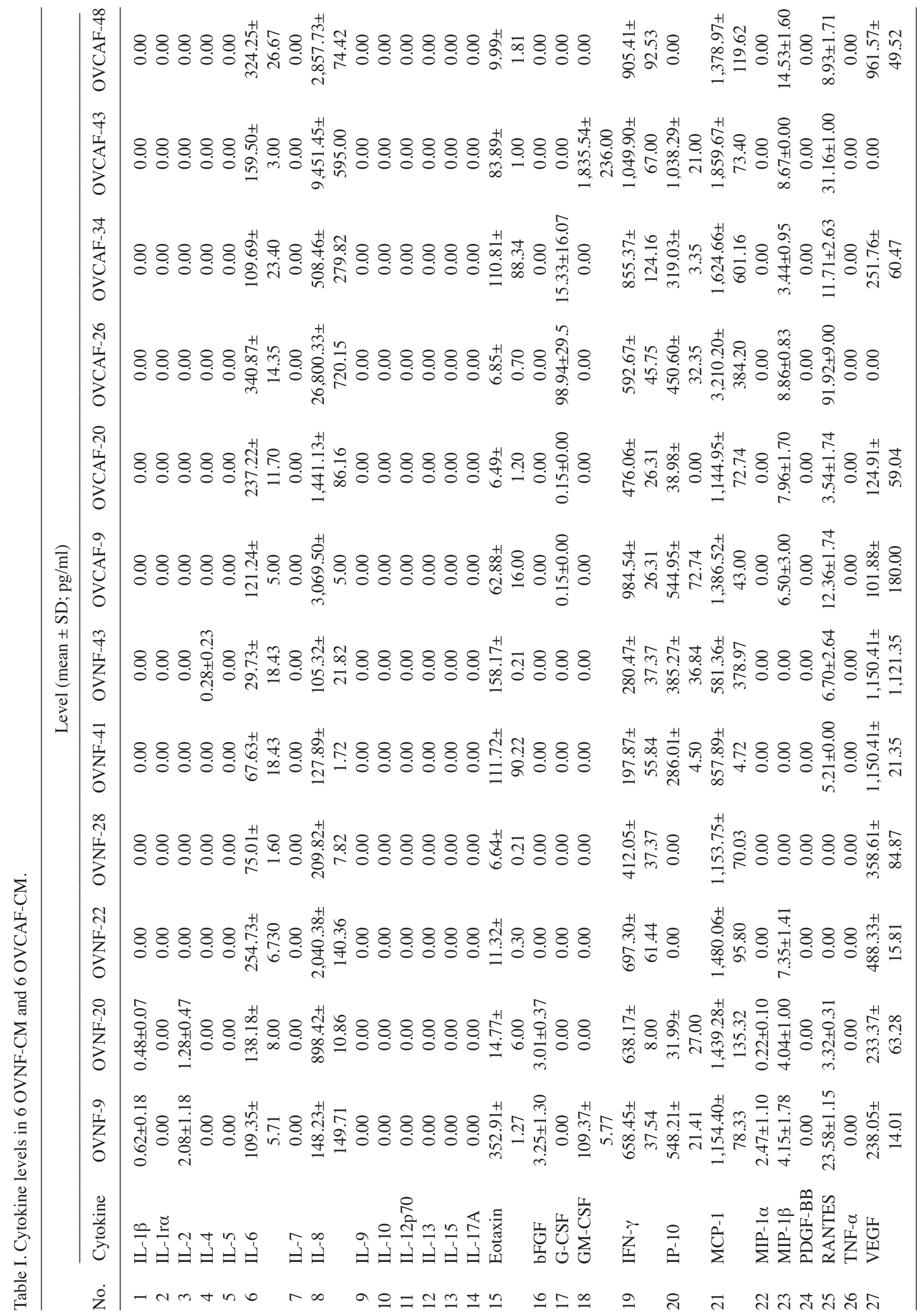


A

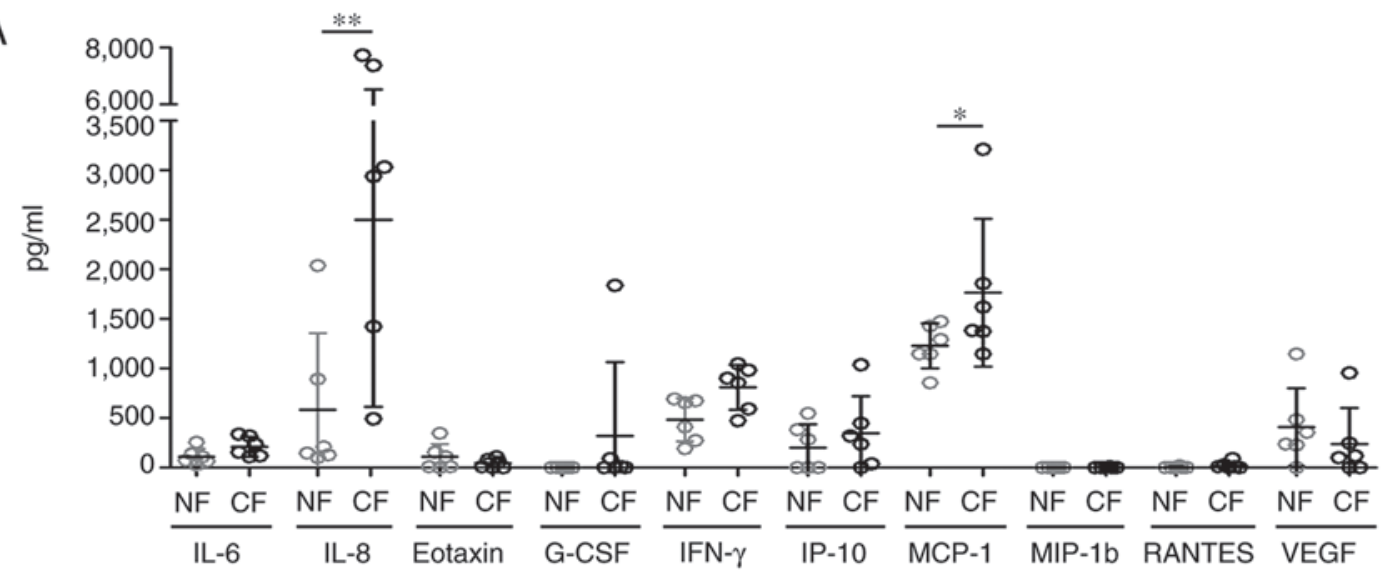

B

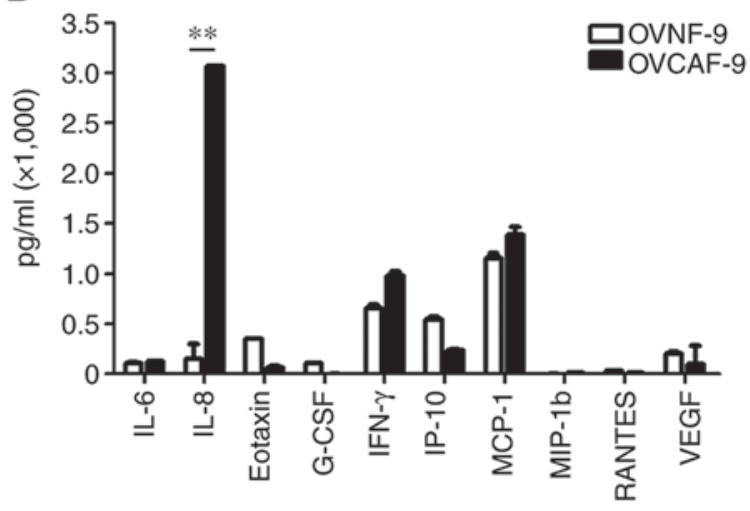

C

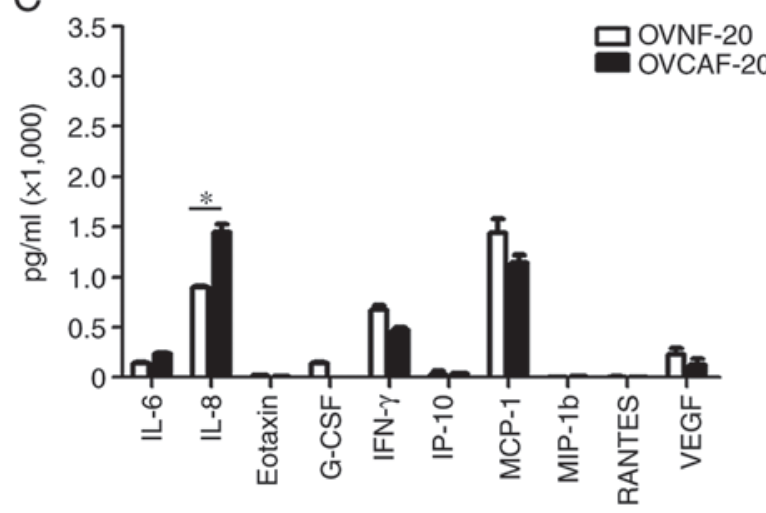

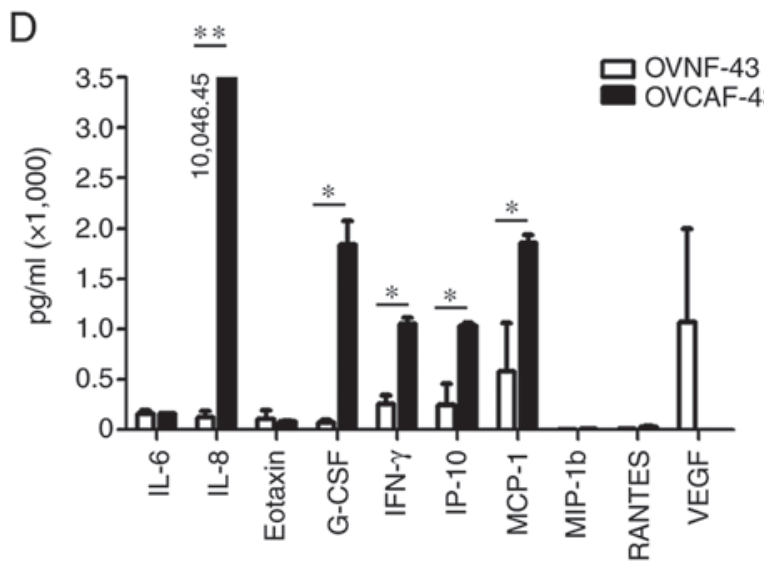

Figure 3. OVCAF-derived substances contain several cytokines/chemokines. (A) Scatter plots present the comparison of secreted substances in 6 pooled cases OVCAFs (CF) and 6 pooled cases OVNFs (NFs), (B) of OVCAF and OVNF (no. 9), (C) of OVCAF and OVNF (no. 20), and (D) of OVCAF and OVNF (no. 43). Data represent the means \pm SD of 3 independent experiments in triplicate. Student's t-test showing the significant differences of OVCAF compared to normal fibroblasts, ${ }^{*} \mathrm{P}<0.05,{ }^{* *} \mathrm{P}<0.01$. OVNFs, ovarian non-tumor-associated fibroblasts; OVCAFs, ovarian cancer-associated fibroblasts.

lane 3 vs. lane 2). In a wound-healing assay, the addition of rhIL-8 markedly promoted wound closure, much alike in the culture with $10 \%$ FBS, supporting the hypothesis that FBS contains IL-8 (Fig. 4F and H; with respective quantification data shown in Fig. 4G and I). The data also demonstrated that the SKOV3 cells were more responsive than the Kuramochi cells to IL-8. Indeed, the Kuramochi cells exhibited less motility and required a longer time (up to $48 \mathrm{~h}$ ) for wound healing in the presence of a high FBS or IL-8 concentration compared to the SKOV3 cells.

Finally, a wound-healing assay was performed using cells incubated with rhIL-8 in the presence of rapamycin or metformin, two drugs known to induce autophagy via mTOR inhibition and AMPK activation, respectively. Both autophagy inducers neutralized the migratory capability of the SKOV3 (Fig. 5A and B) and Kuramochi (Fig. 5C and D) ovarian cancer cells exposed to IL-8. These findings confirm that the pro-migratory effect of IL- 8 is mechanistically linked to the downregulation of autophagy.

OVCAF-CM reduces autophagy and mediates cancer cell migration through $I L-8$. The possible involvement of IL-8 in the modulation of autophagy by FBS and the CM from normal and CAFs was investigated further. To this end, the cellular 
A

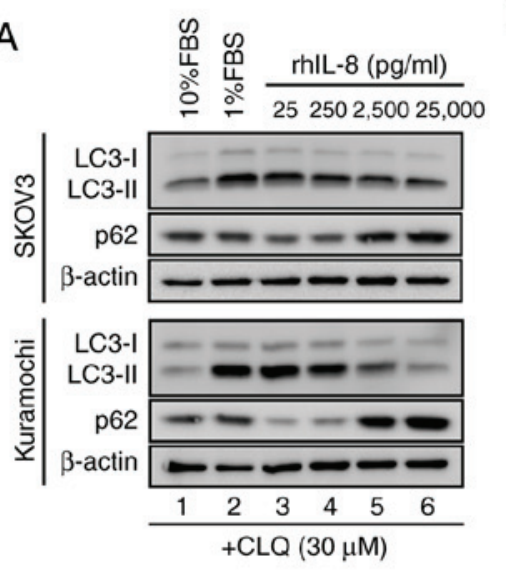

B
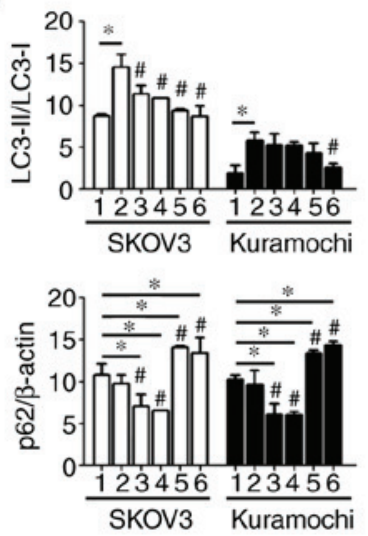

E

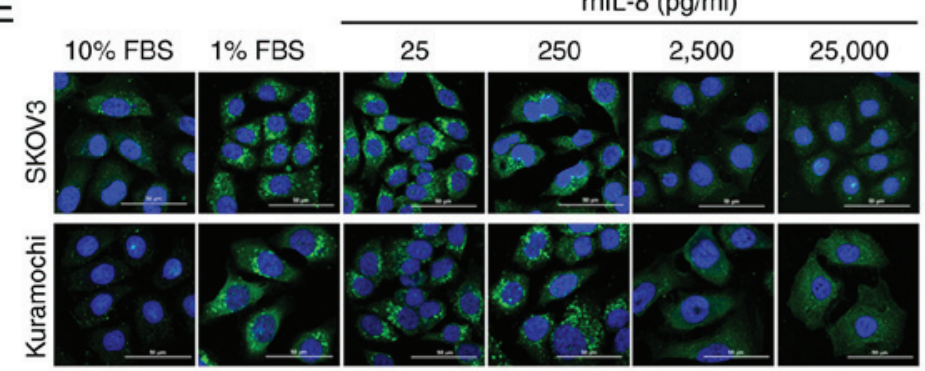

$\mathrm{F}$

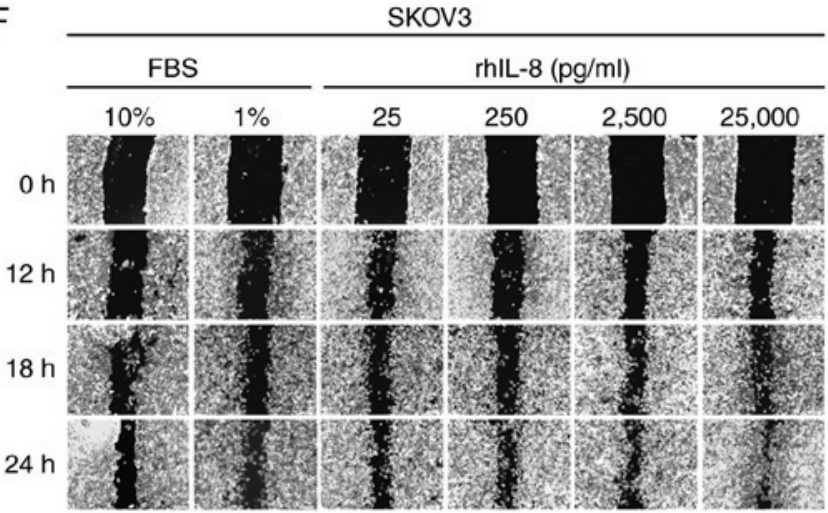

$\mathrm{H}$

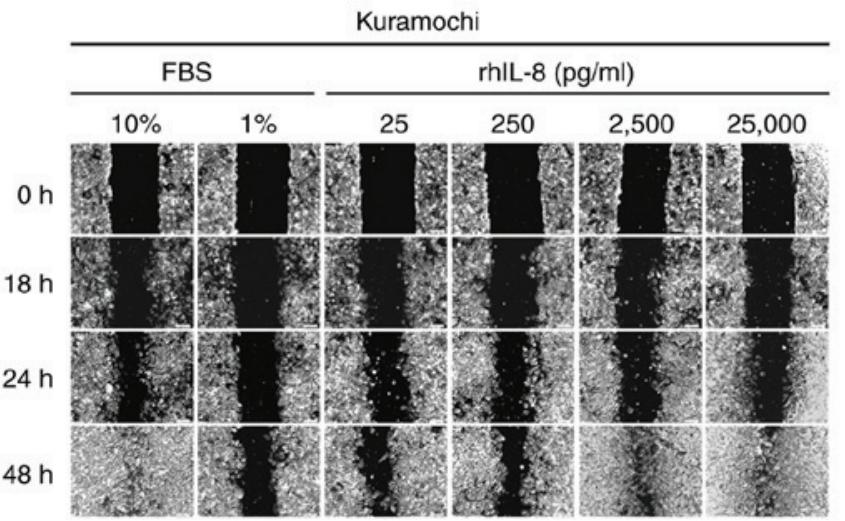

C

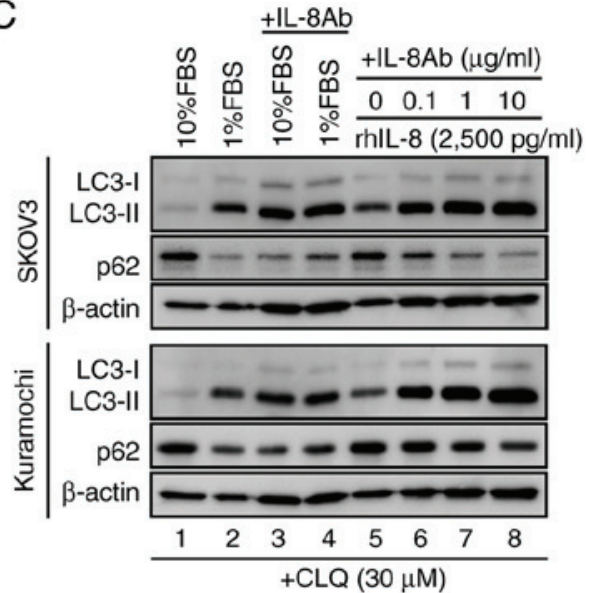

D
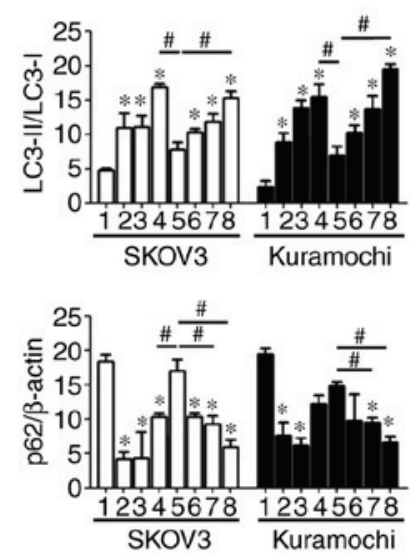

G
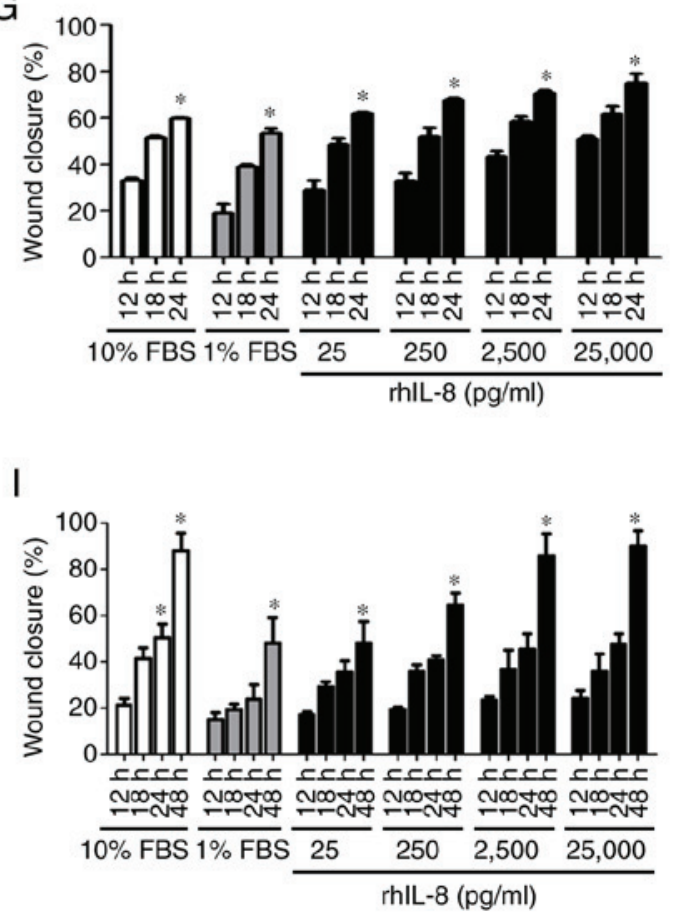

Figure 4. IL-8 and the effect on ovarian cancer cell migration and autophagy. Expression of LC3 in SKOV3 and Kuramochi cells treated or not with rhIL-8 for $24 \mathrm{~h}$ and in the presence of $30 \mu \mathrm{M} \mathrm{CLQ}$ (added $8 \mathrm{~h}$ before cell lysis). (A and B) Representative western blots showing the ratio of LC3-II/LC3-I and p62/ $\beta$-actin of 3 experiments. Densitometry data of 3 separate experiments are reported as the means \pm SD. (C and D) SKOV3 and Kuramochi cells were treated with rhIL-8 $(2,500 \mathrm{pg} / \mathrm{ml})$ either with control or various concentrations of anti-IL-8 antibody and in the presence of $30 \mu \mathrm{M}$ CLQ (added $8 \mathrm{~h}$ before cell lysis). (E) Immunostaining for LC3 (green fluorescence) observed under a fluorescence microscope. Representative images of 2 independent experiments are shown. Nuclei were counterstained with Hoechst. Scale bar, $50 \mu \mathrm{M}$; magnification, x63. (F and $\mathrm{H}$ ) Representative phase-contrast images of the wound healing assay. The cells were incubated in culture medium with 1\%, 10\% FBS and different concentration of rhIL-8 in 10\% FBS medium, as indicated. Images were taken and the width of the wound area was measured at the time indicated. The graphs report the rate of wound closure (\%) for each time point estimated using ImageJ software. (G and I) Data represent the average \pm SD calculated for 3 different fields per each condition in 3 independent experiments. "P<0.05 compared between the indicated values. ${ }^{~} \mathrm{P}<0.05$. CLQ, cholorquine; IL, interleukin; LC3, light chain 3. 
A
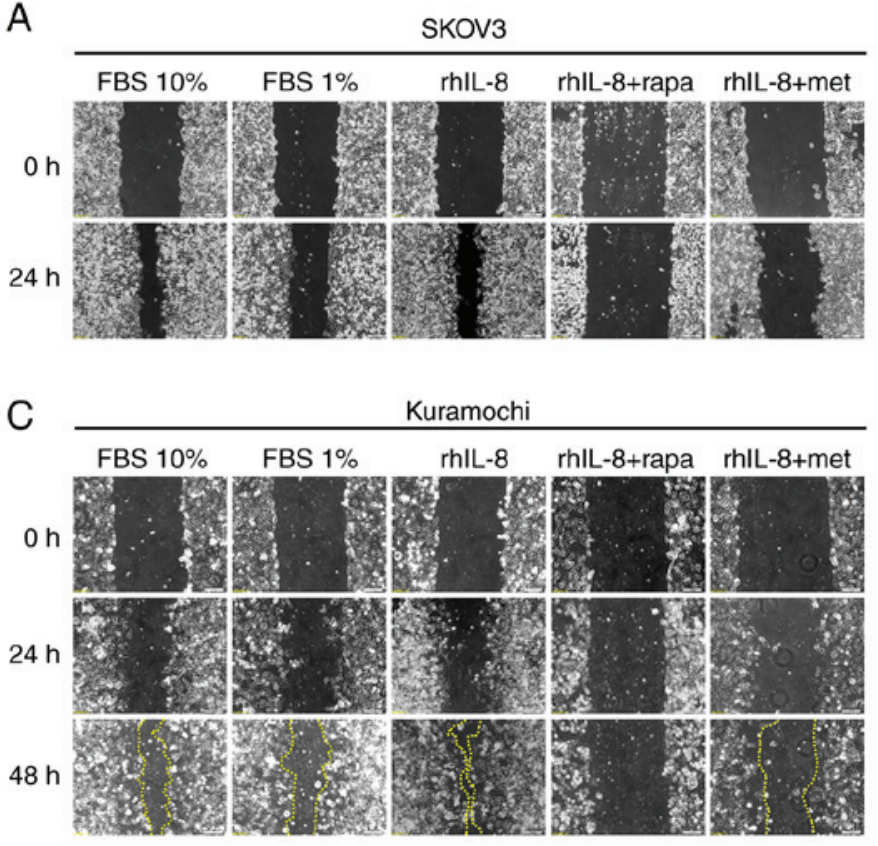

$\mathrm{B}$

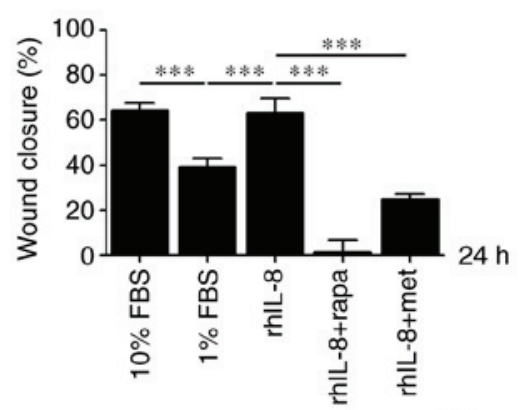

D

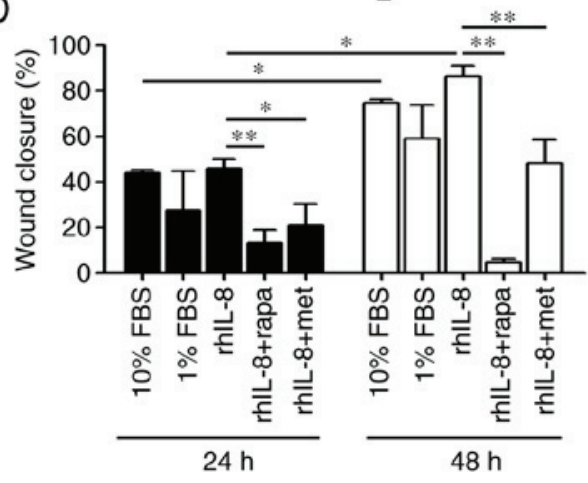

Figure 5. The autophagy inducers, rapamycin and metformin, counteract the promigratory activity of IL- 8 . Representative phase-contrast images of the scratching wound healing assay of (A) SKOV3 and (C) Kuramochi cells. Cells were incubated in $10 \%$ FBS or $1 \%$ FBS medium (respectively as high and low stimulators of cell migration) or in $10 \%$ FBS medium supplemented with $2,500 \mathrm{pg} / \mathrm{ml} \mathrm{rhIL}-8$ without or with $200 \mathrm{nM}$ rapamycin or $2 \mathrm{mM}$ metformin, as indicated. The latter are 2 well-known inducers of autophagy. Images of (B) SKOV3 and (D) Kuramochi cells were acquired and wound closure was measured (as percentage) at time 0,24 and $48 \mathrm{~h}$ using Image J software. Data represent the average \pm SD calculated from 3 different fields per each condition in 3 independent experiments. ${ }^{*} \mathrm{P}<0.05,{ }^{* *} \mathrm{P}<0.01,{ }^{* * * *} \mathrm{P}<0.001$ compared between the indicated values. rhIL-8, recombinant human interleukin 8 .

levels of LC3-II and p62 in the SKOV-3 cells (Fig. 6A-C) and in Kuramochi cells (Fig. 6D-F) cultured in 10 or $1 \%$ FBS or in CM from OVNFs or OVCAFs in the presence of the neutralizing anti-IL-8 antibody was assayed. CLQ was added to prevent autophagosome degradation. Consistent with the data in Fig. 4C and D, in both ovarian cancer cell lines the anti-IL-8 antibody restored autophagy in the cancer cells cultured in $10 \%$ FBS medium to the level found in the control (no anti-IL-8) cells cultured in 1\% FBS medium (Fig. 6A and B, and D and E; lane 3 vs. lane 2), and this effect was statistically significant. Moreover, anti-IL-8 abrogated the negative effect of CAF-CM on autophagy modulation (reduced autophagy), maintaining the LC3-II/LC3-I levels at values comparable to those observed in cells incubated with OVNF-CM or in control 1\% FBS medium (Fig. 6A and B, and $\mathrm{D}$ and $\mathrm{E}$; lanes 5-10 compared to lane 2). A decreased accumulation of p62 was observed in the OVCAF-CM (cases no 20 and 43)-treated cells upon the addition of anti-IL-8 antibody compared to that in the control cells incubated in 10\% FBS medium (Fig. 6A-C, and D-F; lanes 5-10 compared to lane 2), indicating that anti-IL-8 antibody restored the autophagic flux, reverting the block induced by OVCAF-CM.

Finally, to link this observation with the functional effect on cell migration, the expression levels of LC3 and E-cad in the cells at the migration front in the cancer cells cultured as described above were assayed. Neutralizing anti-IL-8 antibody attenuated the migration of both ovarian cancer cells cultured in 10\% FBS medium (Fig. 6G and H). Anti-IL8 antibody increased the expression of LC3 and of E-cad in the cells incubated in $1 \%$ FBS, while this effect was less prominent in the cells cultured in 10\% FBS. Similarly, anti-IL8 antibody blocked the cell migration induced by OVCAFs-CM concomitantly, with the upregulation of LC3 expression (indicative of autophagy) and the downregulation of E-cad expression (indicative of migratory cells) in the cells at the migration front (Fig. 6G and H). From these data, it can be concluded that OVCAF-CM reduces autophagy and mediates cancer cell migration through IL-8.

\section{Discussion}

CAFs in the tumor microenvironment have been well-recognized for their potential in inducing cancer cell progression in ovarian cancer $(3,4)$. Several cytokines/chemokines from ovarian CAFs have been proven to modulate autophagy (3). The migration rate of ovarian cancer cells increases upon autophagy gene knockdown (13). However, to date, to the best of our knowledge, there are no data demonstrating a direct effect of cytokines/chemokines secreted by ovarian cancer CAFs on the modulation of autophagy and how this modulation can affect ovarian cancer cell migration. In the present study, cytokines and chemokines, including IL-6, IL-8, eotaxin, GM-CSF, IFN- $\gamma$, IP-10, MCP-1, MIP-1b, RANTES and VEGF were detected in the conditioned-medium of 3 primary CAFs from ovarian cancer, indicating their possible release in the microenvironment and their participation in ovarian cancer progression. In fact, IFN- $\gamma$ from tumor infiltrating lymphocytes has been shown to regulate the expression of programed death ligand-1 (PD-L1) in ovarian cancer cells (25). Moreover, IFN- $\gamma$, TNF- $\alpha$, IL-10 and IL- 6 released from tumor-associated 
A

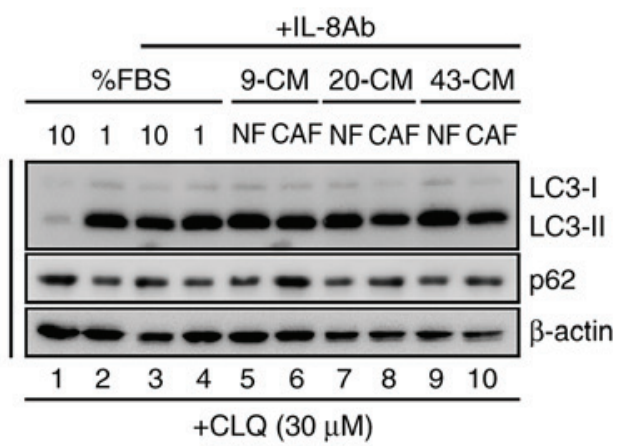

D

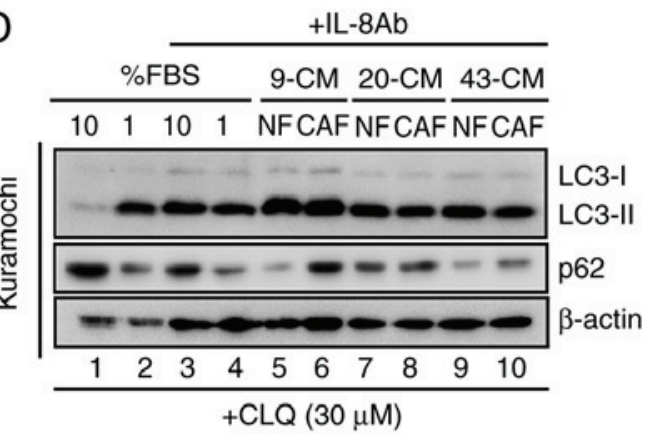

B

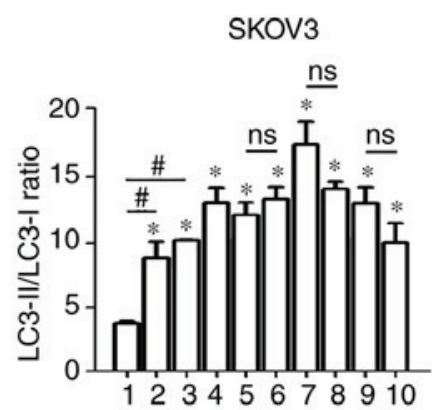

E

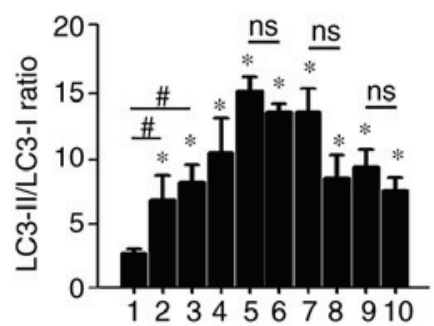

SKOV3
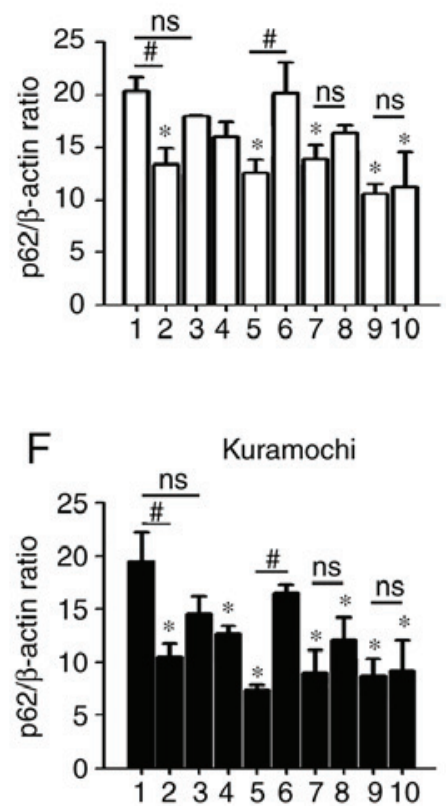

G

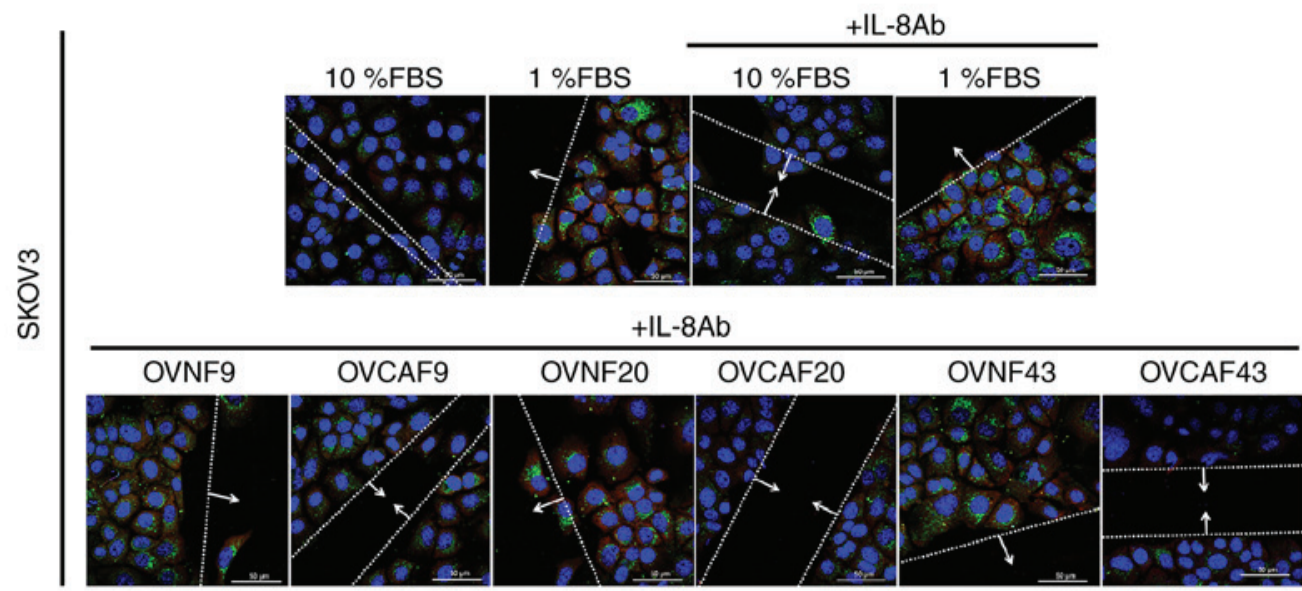

LC3/E-cad/Hoechst

$\mathrm{H}$

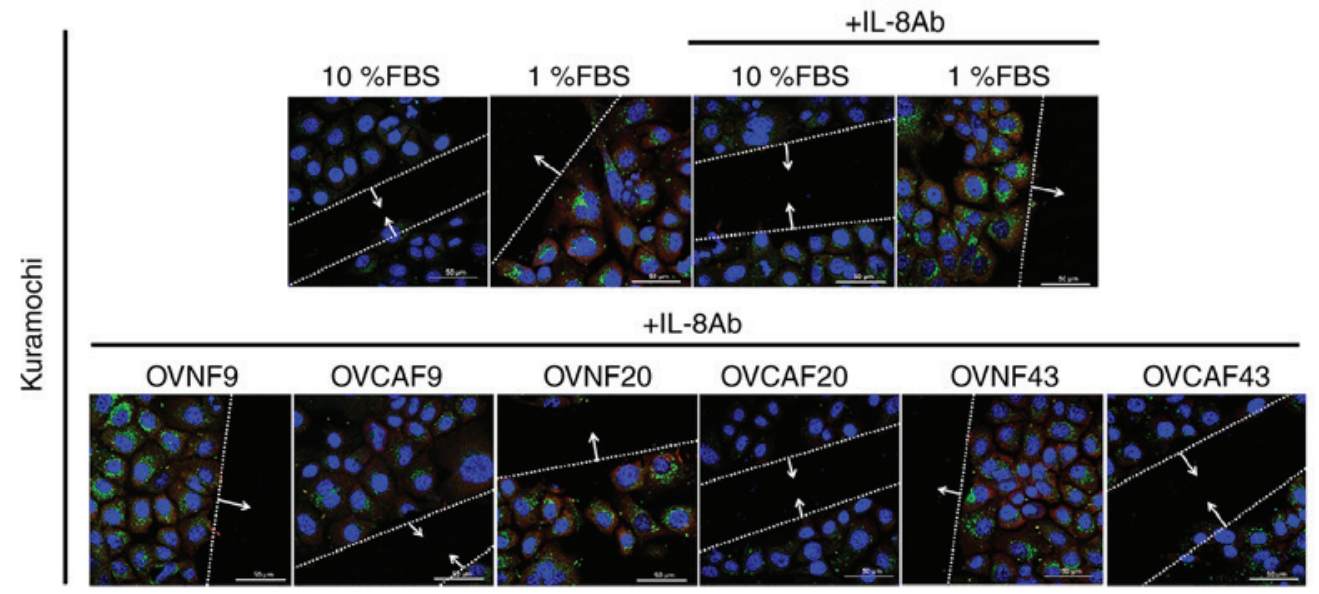

LC3/E-cad/Hoechst

Figure 6. Neutralization of IL-8 can restore OVCV-CM-reduced autophagy of OVC cells and attenuate OVCAF-CM-induced migration. Western blot analysis of LC3 and p62 expression in (A-C) SKOV3 and in (D-F) Kuramochi cells cultured in medium containing 10 or $1 \%$ FBS or in CM from OVNFs or OVCAFs supplemented with anti-IL8 neutralizing antibody as indicated, and in the presence of $30 \mu \mathrm{M}$ CLQ. Immunofluorescence staining of LC3 (green fluorescence) and E-cad (red fluorescence) in the wound area was performed in (G) SKOV3 and (H) Kuramochi cells cultured for $24 \mathrm{~h}$ in OVCAF-CM or OVNF-CM with anti-IL-8 neutralizing antibody. Staining with Hoechst (blue fluorescence) was performed to visualize chromatin. Data indicate that anti-IL-8 restored the expression of LC3 and of E-cad in the cells at the migration front. ${ }^{~} \mathrm{P}<0.05$ compared with control $10 \%$ FBS treatment, ${ }^{*} \mathrm{P}<0.05$; ns, not significant compared between the indicated values. OVNFs, ovarian non-tumor-associated fibroblasts; OVCAFs, ovarian cancer-associated fibroblasts; CLQ, chloroquine. 
macrophages have been shown to stimulate the expression of PD-L1 at the surface of the cancer cells (26). As shown in a previous study, MCP-1 promoted the invasion and adhesion of ovarian cancer cells, and a CCR2 antagonist attenuated the effects of MCP-1 in vitro (27). High concentrations of IP-10 and MCP-1 have been detected in both ascites and tumor cells of ovarian cancer patients (28). This evidence supports the tumorigenic promoting effect of the substances released from CAFs in ovarian cancer.

In the present study, OVCAFs were characterized by the presence of (29). The lack of positivity for the epithelial marker CK19 in CAF culture ensures no contamination by cancer cells. In a previous study, CK19 was found to be highly expressed at the same level of CK7 in three ovarian cancer cells (Caov-3, OVCAR-3 and SKOV3), including the one used in the present study (30). By contrast, CK7 was not expressed in other ovarian cancer cell lines (PA-1 and A2780ADR) that however expressed CK19 (30). Additionally, the upregulation of CK19 has been shown to be associated with the proliferation, migration and invasion of ovarian cancer cells, and is in fact considered a potential therapeutic target $(31,32)$. These data confirm that CK19 is a reliable marker for identifying ovarian cancer cells and support its use for examining epithelial contamination in OVCAF primary culture.

CAFs are activated fibroblasts in the tumor microenvironment and their metastasis-promoting functions has been well-established (33). CAFs can promote the tumor growth by modulating the actual level of autophagy in ovarian cancer cells through the secretion of pro-inflammatory cytokines and the release of autophagy-derived metabolites and substrates (3). Several cytokines, including IFN- $\gamma$, IL-4, IL- 6 and IL-13, have been shown to be associated with autophagy regulation, yet the majority of studies has focused on IL-6, as this is one of the main cytokines released in the tumor microenvironment. Apparently, this cytokine exerts pleiotropic effects on autophagy regulation that likely are cell- and extracellular environment-context dependent. In a previous study, human IL-6 recombinant protein induced autophagy in HepG2 liver cancer cells through the induction of $\mathrm{NF}-\kappa \mathrm{B}$ mediated signaling pathway (34). Yet, other studies have demonstrated an inhibitory effect of IL-6 on autophagy regulation in cancer cells. For instance, IL- 6 has been shown to reduce the expression of LC3-II and BECLIN-1, thus inhibiting autophagy in starved U937 cells through the STAT3 signaling pathway (35). IL- 6 has also been shown to promote cancer cell migration via the inhibition of autophagy. The conditioned-medium from human primary cholangiocarcinoma CAFs, which has been shown to contain high levels of IL-6 and IL-8, cab promote the migration of human cholangiocarcinoma cells through the inhibition of autophagy in cancer cells, and this effect is abolished when the secretion of IL- 6 in the CAFs conditioned medium is prevented (21). With reference to ovarian cancer, it has been shown that IL-6 can promote cell migration through the inhibition of BECLIN-1-dependent autophagy in the cells at the migration front (18). While the role of IL-6 in modulating autophagy and cell migration has been well addressed in ovarian cancer cells and in other types of cancer, little is known about the role played by IL- 8 .

E-cadherin plays a pivotal role in cell-cell adhesion, and it is not expressed on the membrane and it is epigenetically silenced in the cells that disaggregate to begin moving. The switch from $\mathrm{E}$ - to $\mathrm{N}$-cadherin expression on cell surface is a marker of EMT. This is likely to be more evident in the cells that lose the cell-cell contact and start to migrate, as also demonstrated in a previous study by the authors (18), beside others $(7,9)$. On the other hand, it is not expected that all cells would exhibit a downregulation of E-cadherin to the same extent, as indicated by the lack of a typical scatter phenomenon. In fact, cytokines downregulate autophagy and E-cadherin expression preferentially in the cells at the migration front (18). Therefore, western blot analysis of the whole homogenate would provide an average level of reduced expression. For these reasons, it is more informative to examine the expression of E-cadherin on the membrane of the cells at the migration front as a confirmation of their acquired ability to move.

IL-8 has been shown to attenuate the DIRAS3-mediated autophagy (19), induce EMT and enhance ovarian cancer cell metastasis (7,9). Consistently, in vitro analyses have indicated that the overexpression and secretion of IL- 8 in ovarian cancer cells favor their anchorage-independent growth, proliferation and invasion (20). In the present study, IL-8 was found highly expressed in and secreted by OVCAFs compared to normal fibroblasts. The CM from CAFs isolated from 3 primary ovarian cancers were shown to stimulate cell migration in 2 ovarian cancer cell lines, and this effect was parallel to the downregulation of autophagy in the cancer cells. These effects were abolished when the CAFs-CM was supplemented with anti-IL8 neutralizing antibody. Of note, FBS at $10 \%$ elicited a similar effect in promoting cell migration along with the suppression of autophagy. Again, the addition of anti-IL8 neutralizing antibody to the medium with $10 \%$ FBS restored autophagy and blocked cell migration to the level observed in the control cells cultured in 1\% FBS medium, thus suggesting the presence of IL-8 in FBS. Although the involvement of IL-8 in the migration and invasion of ovarian cancer SKOV3 cells has previously been reported (8), to the best of our knowledge, this is the first study to demonstrate that CAF-derived IL-8 negatively regulates autophagy and concomitantly induces the migration of 2 ovarian cancer cell lines. The mechanistic link between the downregulation of autophagy and increased cell migration was definitively proven, showing that the over-stimulation of autophagy with rapamycin and metformin counteracted the promigratory activity of rhIL-8. These results are consistent with previous evidence indicating that the knockdown of autophagy-related genes favors ovarian cancer cell migration (13). IL-8 can negatively regulate autophagy via the activation of the PI3K/AKT and MAPK/ERK signaling pathways (19).

In conclusion, the present study demonstrates that CAF-derived IL-8 negatively regulates autophagy and concomitantly induces ovarian cancer cell migration. The findings presented herein indicate that antibody targeting IL- 8 released by CAFs and other stromal cells may prove to be an additional therapeutic tool with which to limit ovarian cancer progression.

\section{Acknowledgements}

The authors would like to thank Miss Vijakhana Pilaisangsuree, Mahidol University for providing technical support. The authors would also like to thank Miss Marisa Royrod, Mahidol 
University for providing technical assistance with autophagy detection.

\section{Funding}

The present study was supported by the Siriraj Cancer Foundation, Siriraj Hospital, Mahidol University and Research Grants from Siriraj Research Division (R016033015) to CT. AF is recipient of a post-doctoral fellowship 'Paolina Troiano' (id. 24094) granted by Associazione Italiana per la Ricerca sul Cancro (AIRC, Milan, Italy).

\section{Availability of data and materials}

All data generated or analyzed during this study are included in this published article or are available from the corresponding author on reasonable request.

\section{Authors' contributions}

CT, PT and CI designed the experiments. SThongchot, PJ, STherasakvichya, MW and AF performed the experiments, elaborated the data, performed the quantification and statistical analysis, and prepared the figures. PT, CT and CI interpreted the results. SThongchot, PT and CT drafted the manuscript. CT and CI revised and finalized the manuscript. All authors read and approved the final manuscript.

\section{Ethics approval and consent to participate}

Not applicable.

\section{Patient consent for publication}

Not applicable.

\section{Competing interests}

Authors declare that they have no competing interests.

\section{References}

1. Ahmed N, Kadife E, Raza A, Short M, Jubinsky PT and Kannourakis G: Ovarian cancer, cancer stem cells and current treatment strategies: A potential role of magmas in the current treatment methods. Cells 9: 719, 2020.

2. Jayson GC, Kohn EC, Kitchener HC and Ledermann JA: Ovarian cancer. Lancet 384: 1376-1388, 2014.

3. Thuwajit C, Ferraresi A, Titone R, Thuwajit P and Isidoro C: The metabolic cross-talk between epithelial cancer cells and stromal fibroblasts in ovarian cancer progression: Autophagy plays a role. Med Res Rev 38: 1235-1254, 2018.

4. Jiang Y, Wang C and Zhou S: Targeting tumor microenvironment in ovarian cancer: Premise and promise. Biochim Biophys Acta Rev Cancer 1873: 188361, 2020.

5. Zhang L, Liu W, Wang X, Wang X and Sun H: Prognostic value of serum IL-8 and IL-10 in patients with ovarian cancer undergoing chemotherapy. Oncol Lett 17: 2365-2369, 2019.

6. Rodrigues ISS, Martins-Filho A, Micheli DC, Lima CA Tavares-Murta BM, Murta EFC and Nomelini RS: IL-6 and IL-8 as prognostic factors in peritoneal fluid of ovarian cancer Immunol Invest 49: 510-521, 2020.

7. Wen J, Zhao Z, Huang L, Wang L, Miao Y and Wu J: IL-8 promotes cell migration through regulating EMT by activating the Wnt/ $\beta$-catenin pathway in ovarian cancer. J Cell Mol Med 24: $1588-1598,2020$.
8. Li Y, Liu L, Yin Z, Xu H, Li S, Tao W, Cheng H, Du L, Zhou X and Zhang B: Effect of targeted silencing of IL-8 on in vitro migration and invasion of SKOV3 ovarian cancer cells. Oncol Lett 13: 567-572, 2017.

9. Yin J, Zeng F, Wu N, Kang K, Yang Z and Yang H: Interleukin-8 promotes human ovarian cancer cell migration by epithelial-mesenchymal transition induction in vitro. Clin Transl Oncol 17: 365-370, 2015.

10. Uddin MM, Gaire B and Vancurova I: Interleukin- 8 induces proliferation of ovarian cancer cells in 3D spheroids. Methods Mol Biol 2108: 117-124, 2020.

11. Ferraresi A, Girone C,Esposito A, Vidoni C, Vallino L, SecomandiE, Dhanasekaran DN and Isidoro C: How autophagy shapes the tumor microenvironment in ovarian cancer. Front Oncol 10: 599915, 2020.

12. Peracchio C, Alabiso O, Valente $G$ and Isidoro C: Involvement of autophagy in ovarian cancer: A working hypothesis. J Ovarian Res 5: 22, 2012.

13. Delaney JR, Patel CB, Bapat J, Jones CM, Ramos-Zapatero M, Ortell KK, Tanios R, Haghighiabyaneh M, Axelrod J, DeStefano JW, et al: Autophagy gene haploinsufficiency drives chromosome instability, increases migration, and promotes early ovarian tumors. PLoS Genet 16: e1008558, 2020.

14. Cai M, Hu Z, Liu J, Gao J, Liu C, Liu D, Tan M, Zhang D and Lin B: Beclin 1 expression in ovarian tissues and its effects on ovarian cancer prognosis. Int J Mol Sci 15: 5292-5303, 2014.

15. Zhao Y, Chen S, Gou WF, Xiao LJ, Takano Y and Zheng HC: Aberrant Beclin 1 expression is closely linked to carcinogenesis, differentiation, progression, and prognosis of ovarian epithelial carcinoma. Tumour Biol 35: 1955-1964, 2014.

16. Valente G, Morani F, Nicotra G, Fusco N, Peracchio C, Titone R, Alabiso O, Arisio R, Katsaros D, Benedetto C and Isidoro C: Expression and clinical significance of the autophagy proteins BECLIN 1 and LC3 in ovarian cancer. Biomed Res Int 2014: 462658, 2014

17. Spowart JE, Townsend KN, Huwait H, Eshragh S, West NR, Ries JN, Kalloger S, Anglesio M, Gorski SM, Watson PH, et al: The autophagy protein LC3A correlates with hypoxia and is a prognostic marker of patient survival in clear cell ovarian cancer. J Pathol 228: 437-447, 2012.

18. Ferraresi A, Phadngam S, Morani F, Galetto A, Alabiso O, Chiorino $\mathrm{G}$ and Isidoro $\mathrm{C}$ : Resveratrol inhibits IL-6-induced ovarian cancer cell migration through epigenetic up-regulation of autophagy. Mol Carcinog 56: 1164-1181, 2017.

19. Mao W, Peters HL, Sutton MN, Orozco AF, Pang L, Yang H, $\mathrm{Lu} \mathrm{Z}$ and Bast RC Jr: The role of vascular endothelial growth factor, interleukin 8, and insulinlike growth factor in sustaining autophagic DIRAS3-induced dormant ovarian cancer xenografts. Cancer 125: 1267-1280, 2019.

20. Wang Y, Xu RC, Zhang XL, Niu XL, Qu Y, Li LZ and Meng XY: Interleukin-8 secretion by ovarian cancer cells increases anchorage-independent growth, proliferation, angiogenic potential, adhesion and invasion. Cytokine 59: 145-155, 2012.

21. Thongchot S, Ferraresi A, Vidoni C, Loilome W, Yongvanit P, Namwat $\mathrm{N}$ and Isidoro $\mathrm{C}$ : Resveratrol interrupts the pro-invasive communication between cancer associated fibroblasts and cholangiocarcinoma cells. Cancer Lett 430: 160-171, 2018.

22. Heger JI, Froehlich K, Pastuschek J, Schmidt A, Baer C, Mrowka R, Backsch C, Schleußner E, Markert UR and Schmidt A: Human serum alters cell culture behavior and improves spheroid formation in comparison to fetal bovine serum. Exp Cell Res 365: 57-65, 2018.

23. Li B, Sun C, Sun J, Yang MH, Zuo R, Liu C, Lan WR, Liu MH, Huang B and Zhou Y: Autophagy mediates serum starvationinduced quiescence in nucleus pulposus stem cells by the regulation of P27. Stem Cell Res Ther 10: 118, 2019.

24. Klionsky DJ, Abdelmohsen K, Abe A, Abedin MJ, Abeliovich H, Acevedo Arozena A, Adachi H, Adams CM, Adams PD, Adeli K, et al: Guidelines for the use and interpretation of assays for monitoring autophagy (3rd edition). Autophagy 12: 1-222, 2016.

25. Abiko K, Matsumura N, Hamanishi J, Horikawa N, Murakami R, Yamaguchi K, Yoshioka Y, Baba T, Konishi I and Mandai M: IFN- $\gamma$ from lymphocytes induces PD-L1 expression and promotes progression of ovarian cancer. Br J Cancer 112: 1501-1509, 2015.

26. Qu QX, Xie F, Huang Q and Zhang XG: Membranous and cytoplasmic expression of PD-L1 in ovarian cancer cells. Cell Physiol Biochem 43: 1893-1906, 2017.

27. Furukawa S, Soeda S, Kiko Y, Suzuki O, Hashimoto Y, Watanabe T, Nishiyama H, Tasaki K, Hojo H, Abe $M$ and Fujimori K: MCP-1 promotes invasion and adhesion of human ovarian cancer cells. Anticancer Res 33: 4785-4790, 2013. 
28. Rådestad E, Klynning C, Stikvoort A, Mogensen O, Nava S Magalhaes I and Uhlin M: Immune profiling and identification of prognostic immune-related risk factors in human ovarian cancer. Oncoimmunology 8: e1535730, 2019.

29. Lai D, Ma L and Wang F: Fibroblast activation protein regulates tumor-associated fibroblasts and epithelial ovarian cancer cells. Int J Oncol 41: 541-550, 2012.

30. Stimpfl M, Schmid BC, Schiebel I, Tong D, Leodolter S, Obermair A and Zeillinger R: Expression of mucins and cytokeratins in ovarian cancer cell lines. Cancer Lett 145: 133-141, 1999.

31. Wu HH, Wang PH, Yeh JY, Chen YJ, Yen MS, Huang RL, Tsai YJ and Yuan CC: Serum cytokeratin-19 fragment (Cyfra 21-1) is a prognostic indicator for epithelial ovarian cancer. Taiwan $\mathrm{J}$ Obstet Gynecol 53: 30-34, 2014.

32. Lu Q, Qu H, Lou T, Liu C and Zhang Z: CK19 promotes ovarian cancer development by impacting on Wnt/ $/$-catenin pathway. Onco Targets Ther 13: 2421-2431, 2020.
33. Kwa MQ, Herum KM and Brakebusch C: Cancer-associated fibroblasts: How do they contribute to metastasis? Clin Exp Metastasis 36: 71-86, 2019.

34. Lu H, Han M, Yuan X, Tursun K, Zhang Y, Li Y, Li Z, Feng S, Zhou L, Pan Z, et al: Role of IL-6-mediated expression of NS5ATP9 in autophagy of liver cancer cells. J Cell Physiol 233: 9312-9319, 2018.

35. Qin B, Zhou Z, He J, Yan C and Ding S: IL-6 inhibits starvation-induced autophagy via the STAT3/Bcl-2 signaling pathway. Sci Rep 5: 15701, 2015.

This work is licensed under a Creative Commons Attribution-NonCommercial-NoDerivatives 4.0 International (CC BY-NC-ND 4.0) License. 